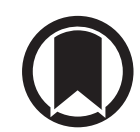

CrossMark

\title{
Applicability of the World Health Organization recommended new shorter regimen in a multidrug-resistant tuberculosis high burden country
}

\begin{abstract}
To the Editor:
In order to improve the adherence and treatment outcomes of multidrug-resistant (MDR) tuberculosis (TB) patients, the World Health Organization (WHO) has recently recommended a new, shorter and cheaper treatment regimen. The new regimen, comprised of 4-6 months of kanamycin, moxifloxacin, prothionamide, clofazimine, pyrazinamide, ethambutol and high dose isoniazid followed by 5 months of moxifloxacin, clofazimine, pyrazinamide and ethambutol [1], has produced excellent outcomes under operational research conditions is various settings [2-4]. However, the recommendation that it should be used only for MDR-TB patients who are neither previously treated with second-line anti-TB drugs, nor resistant to fluoroquinolones or injectable second-line anti-TB drugs [1], generates concerns about its applicability in MDR-TB high burden countries [5]. In the current cross-sectional study, culture-confirmed MDR-TB patients treated at two units for programmatic management of drug-resistant TB in Pakistan were evaluated for drug resistance pattern and eligibility to be treated with the new shorter regimen.
\end{abstract}

A total of 832 culture-confirmed MDR-TB patients consecutively enrolled for treatment at Lady Reading Hospital Peshawar (Khyber-Pukhtoonkhwa) and Nishtar Hospital Multan (Punjab) from January 2012 to July 2016 met the inclusion criteria and were included in the study. Patients with drug-resistant TB other than MDR-TB (extensively drug-resistant, poly-drug-resistant and mono-drug-resistant TB) and who had a history of MDR-TB treatment were excluded. At the study sites, presumed drug-resistant TB patients were initially evaluated with two sputum samples for acid-fast bacilli by direct sputum smear microscopy using Ziehl Neelsen staining method and GeneXpert System's MTB/Rif (Mycobacterium tuberculosis/rifampicin). Upon positive smear microscopy and rapid drug susceptibility test (DST), sputum samples were sent to Aga Khan University Hospital Laboratory, Karachi, Pakistan, National TB reference laboratory, Islamabad, Pakistan or Provincial TB reference laboratory, Peshawar, Pakistan for sputum culture and DST. At the reference laboratories, DST was carried out by using Agar proportion method on enriched Middlebrook 7H10 medium (BBL; Beckton Dickinson, Sparks, MD, USA) at the following concentrations [6]: rifampicin $\left(1 \mu \mathrm{g} \cdot \mathrm{mL}^{-1}\right)$, isoniazid $\left(0.2 \mu \mathrm{g} \cdot \mathrm{mL}^{-1}\right)$, streptomycin $\left(2 \mu \mathrm{g} \cdot \mathrm{mL}^{-1}\right)$, ethambutol $\left(5 \mu \mathrm{g} \cdot \mathrm{mL}^{-1}\right)$, ofloxacin $\left(2 \mu \mathrm{g} \cdot \mathrm{mL}^{-1}\right)$, amikacin $\left(4 \mu \mathrm{g} \cdot \mathrm{mL}^{-1}\right)$, kanamycin $\left(5 \mu \mathrm{g} \cdot \mathrm{mL}^{-1}\right)$, capreomycin $\left(4 \mu \mathrm{g} \cdot \mathrm{mL}^{-1}\right)$, and ethionamide $\left(5 \mu \mathrm{g} \cdot \mathrm{mL}^{-1}\right)$. DST for pyrazinamide was carried out at $100 \mu \mathrm{g} \cdot \mathrm{mL}^{-1}$ by using BACTEC Mycobacterial Growth Indicator Tube (MGIT) in accordance with manufacturer's instructions. A standardised data collection form was used to retrospectively abstract patient's information. This study was approved by the research and ethics committee of the Postgraduate Medical Institute, Peshawar, Pakistan.

The majority of patients were female (52.4\%), 21-40 years old (47.5\%) and previously been treated for TB (93.6\%). A high proportion of patients (47.6\%) were resistant to all five first-line anti-TB drugs, and 51.4\% were resistant to second-line anti-TB drugs. Among first-line drugs, resistance was highest for pyrazinamide $(79.6 \%)$, followed by ethambutol $(76.2 \%)$ and streptomycin $(62.6 \%)$. Of second-line drugs, resistance was highest for ofloxacin (48.6\%), followed by ethionamide $(5.6 \%)$ and injectable second-line drugs $(0.4 \%)$. Upon taking resistance to fluoroquinolones (irrespective of generation) as one of the criteria for a patient's ineligibility to be treated with the shorter regimen, a total of $409(49.2 \%)$ study participants

@ERSpublications

High prevalence of ofloxacin resistance should not limit the applicability of the new shorter regimen in Pakistan http://ow.ly/HCAs306dUjU

Cite this article as: Javaid A, Ahmad N, Khan A, et al. Applicability of the World Health Organization recommended new shorter regimen in a multidrug-resistant tuberculosis high burden country. Eur Respir J 2017; 49: 1601967 [https://doi.org/10.1183/13993003.01967-2016]. 
were not eligible for treatment with the shorter regimen, mainly due to ofloxacin resistance (48.6\%) followed by history of second-line anti-TB drug use (1.3\%) and resistance to injectable second-line drugs $(0.4 \%)$. However, a recently conducted multicountry study among TB patients has reported a low level of moxifloxacin resistance in Pakistan. The study reports that at $0.5 \mu \mathrm{g} \cdot \mathrm{mL}^{-1}$, only $7.5 \%$ of new TB cases and $13.8 \%$ of rifmapicin-resistant TB cases in Pakistan were resistant to moxifloxacin, which decreased further to $0.4 \%$ and $1.4 \%$ respectively when tested at $2 \mu \mathrm{g} \cdot \mathrm{mL}^{-1}$. Moreover, when the minimum inhibitory concentration (MIC) for moxifloxacin was increased from $0.5 \mu \mathrm{g} \cdot \mathrm{mL}^{-1}$ to the clinical break point of $2 \mu \mathrm{g} \cdot \mathrm{mL}^{-1}$, the cross-resistance between ofloxacin and moxifloxacin drastically dropped from $72 \%$ to only 7\% [7]. This suggests that resistance to ofloxacin does not necessarily preclude susceptibility to moxifloxacin/gatifloxacin and should not be a reason for depriving MDR-TB patients from the moxifloxacin/gatifloxacin-containing shorter regimen. So in an MDR-TB high burden country like Pakistan, where DST for fluoroquinolones is conducted for ofloxacin only, the applicability of the new regimen can be increased by 1) increasing the MIC for ofloxacin from $2 \mu \mathrm{g} \cdot \mathrm{mL}^{-1}$ to $4 \mu \mathrm{g} \cdot \mathrm{mL}^{-1}$, 2) administration of moxifloxacin at a higher dose of $\geqslant 600 \mathrm{mg}$ once daily and 3) clearing the ambiguity in guideline recommendations [8] by specifically mentioning "resistance to moxifloxacin/gatifloxacin" rather

TABLE 1 Predictors of ofloxacin resistance

\begin{tabular}{|c|c|c|c|c|c|c|}
\hline \multirow[t]{2}{*}{ Variables } & \multicolumn{2}{|c|}{ Ofloxacin resistance } & \multirow[t]{2}{*}{ Univariate analysis } & \multirow[t]{2}{*}{ p-value } & \multirow[t]{2}{*}{ Multivariate analysis } & \multirow[t]{2}{*}{ p-value } \\
\hline & Yes & No & & & & \\
\hline
\end{tabular}

Sex Female

Male

Age years

$\leqslant 20$

$21-40$

$41-60$

$>60$

Weight kg

$<40$

$\geqslant 40$

Residence

Rural

Urban

Smear grading

Negative

Scanty (1-9 AFB per $100 \mathrm{HPF}$ )

+1 (10-99 AFB per $100 \mathrm{HPF}$ )

+2 (1-9 AFB per HPF)

+3 ( $>9$ AFB per HPF)

Previous treatment centre

Public

Private

Public/private matrix

New patients

Previous TB regimen

Category I

Category II

New patients

Unknown

Registration category

New

Failure of previous TB treatment

Relapse

Defaulter of previous TB treatment

Unknown outcome of previous episode

MDR-TB treatment centre

Lady Reading Hospital Peshawar

Nishtar Hospital, Multan
207 (47.5)

$197(49.7)$

$101(48.6)$

$193(48.9)$

$83(46.4)$

27 (54)

$128(50.4)$

$276(47.8)$

290 (50.9)

$130(49.6)$

$22(44.9)$

5 (33.3)

132 (53.2)

102 (39.5)

143 (54.6)

$166(43.1)$

$193(55.5)$

26 (57.8)

19 (35.2)

195 (49.9)

$161(46.3)$

$16(34.8)$

32 (68.1)

$20(36.4)$

$182(46.2)$

27 (45.8)

8 (53.3)

167 (54)

230 (48.3)

174 (48.9)
229 (52.5)

199 (50.3)

107 (51.4)

$202(51.1)$

$96(53.6)$

$23(46)$

$126(49.6)$

302 (52.2)

$280(49.1)$

132 (50.4)

27 (55.1)

10 (66.7)

116 (46.8)

156 (60.5)

$119(45.4)$

219 (56.9)

155 (44.5)

19 (42.2)

35 (64.8)

196 (50.1)

187 (53.7)

30 (65.2)

15 (31.9)

35 (63.6)

$212(53.8)$

32 (54.2)

7 (46.7)

$142(46)$

246 (51.7)

$182(51.1)$
Referent

1.095 (0.834.1438)

0.513

Referent

$1.012(0.723-1.416) \quad 0.944$

$0.916(0.614-1.367) \quad 0.667$

$1.244(0.670-2.310) \quad 0.490$

Referent

$0.900(0.670-1.209) \quad 0.482$

Referent

$0.891(0.450-1.032) \quad 0.452$

Referent

$0.614(0.183-2.062) \quad 0.430$

$1.397(0.754-2.585) \quad 0.288$

$0.802(0.434-1.485) \quad 0.484$

$1.475(0.799-2.723) \quad 0.214$

Referent

$1.643(1.227-2.200)$

$1.805(0.966-3.373)$

$0.716(0.395-1.297)$

0.001

0.064

0.271

Referent

$0.865(0.648-1.156)$

$0.536(0.283-1.015)$

0.327

0.056

$2.144(1.126-4.085) \quad 0.020$

\section{Referent}

$1.502(0.838-2.694)$

$1.477(0.697-3.129)$

$2.000(0.631-6.339)$

0.172

0.309

0.239

0.017

Referent

1.023 (0.777-1.346)
$0.575(0.168-1.693)$

$1.297(0.695-2.421)$

$0.772(0.413-1.443)$

$1.378(0.740-2.566)$

0.377

0.414

0.418

0.313

Referent

$1.531(1.064-2.203)$

$1.668(0.882-3.154)$

$0.487(0.090-2.634)$

0.022

0.115

0.403

Referent

0.910 (0.676-1.225)

$1.051(0.218-5.059)$

$2.087(1.036-4.205)$

0.535

0.951

0.039

Referent

$0.757(0.130-4.424)$

$0.771(0.123-4.813)$

$0.850(0.112-6.465)$

0.757

0.780

0.876

$0.734(0.126-4.294)$

Data are presented as $\mathrm{n}(\%)$ or odds ratios with $95 \%$ confidence intervals, unless otherwise stated. AFB: acid fast bacilli; HPF: high power field;

TB: tuberculosis; MDR: multidrug-resistant. 
than "resistance to fluoroquinolones" as an ineligibility criteria for treatment with the shorter regimen. Furthermore, the high prevalence of cross-resistance between ofloxacin and levofloxacin (87\%) observed in five TB high burden countries [7] advocates for the use of moxifloxacin-containing regimen for MDR-TB treatment rather than the currently used levofloxacin one [9]. Although in the current study resistance to first-line anti-TB drugs which make part of the shorter regimen (pyrazinamide and ethambutol) was highly prevalent, owing to the unrealible nature of DST for these agents, it is not recommended to base decision-making on the basis of resistance to pyrazinamide and ethambutol [10]. Nevertheless, prospective case-controlled studies are required to evaluate the suitability and impact of the new shorter regimen in all geographical regions where MDR-TB is endemic [5] and the prevalence of resistance to second-line drugs is high. As a high rate of ofloxacin resistance was observed in the current study, we evaluated the study participants for the risk factors for ofloxacin resistance. In multivariate analysis, previous TB treatment with unknown regimen (OR 2.087; $\mathrm{p}=0.039$ ) and episode of previous TB treatment in the private sector (OR 1.531; $\mathrm{p}=0.022$ ) emerged as risk factors for ofloxacin resistance (table 1). As resistance to fluoroquinolones has widely been reported as a risk factor for unsuccessful treatment outcomes in MDR-TB patients $[11,12]$, the high degree of ofloxacin resistance among MDR-TB patients in Pakistan $[13,14]$ makes it a threatening issue that could be multifactorial. Patient's previous exposure to fluoroquinolones due to self-medication, over the counter availability of antibiotics in the country and irrational prescription of fluoroquinolones by inadequately trained practitioners upon misdiagnosing $\mathrm{TB}$ with other lower respiratory tract infections are the possible reasons for high fluoroquinolone resistance among MDR-TB patients in Pakistan $[13,14]$. The current finding of previous TB treatment in the private sector as a predictor of ofloxacin resistance is in compliance with a previous report from Pakistan [14]. The inadequate knowledge of private practitioners regarding TB diagnosis and management, divergence from practice guidelines, lack of supervision on patient adherence and complacency regarding patient loss to follow-up have previously been reported from Pakistan $[14,15]$. In the current study, patients with unknown regimen of previous episode of TB treatment were significantly more likely to be ofloxacin resistant. All these patients with the unknown previous regimen had received treatment in the private sector. We suppose that the previous TB treatment with guideline-divergent regimens containing a fluoroquinolone resulted in the emergence of fluoroquinolone resistance in these patients. Despite availability of the free TB treatment in public health facilities through the national TB programme, previous TB treatment in the private health sector of $41.8 \%$ of the current study participants is a finding of concern, especially when there are reports of TB mismanagement in the private sector [14, 15] and its association with high incidence of ofloxacin resistance [14]. This emphasises that national TB programmes should urgently consider innovative ways to make the public health sector more appealing and trustworthy, and train private practitioners in TB management. The concerned health authorities should adopt more restrictive policies to control non-prescription sale of fluoroquinolones, scale up the efforts to improve public awareness about TB and the hazards of self-medication with antibiotics. As the present study included patients from a widely distributed geographical area of two different provinces of Pakistan, we believe that our findings reasonably reflect the drug resistance pattern of MDR-TB patients at other units for programmatic management of drug-resistant TB in the country.

Arshad Javaid ${ }^{1}$, Nafees Ahmad ${ }^{2,3}$, Amer Hayat Khan ${ }^{2}$ and Zubair Shaheen ${ }^{4}$

${ }^{1}$ Dept of Pulmonology, Lady Reading Hospital Peshawar, Peshawar, Pakistan. ${ }^{2}$ Discipline of Clinical Pharmacy, School of Pharmaceutical Sciences, Universiti Sains Malaysia Pulau Pinang, Penang, Malaysia. ${ }^{3}$ Faculty of Pharmacy and Health Sciences, University of Baluchistan, Quetta, Pakistan. ${ }^{4}$ Dept of Pulmonology, Nishtar Hospital, Multan, Pakistan.

Correspondence: Nafees Ahmad, Discipline of Clinical Pharmacy, School of Pharmaceutical Sciences, Universiti Sains Malaysia, Pulau Pinang, Malaysia. E-mail: nafeesuob@gmail.com

Received: Oct 062016 | Accepted after revision: Nov 062016

Conflict of interest: None declared.

Acknowledgements: The authors acknowledge the record keeping staff at the study sites for their help in conducting this study, and have no financial support to report and declare no conflict of interest.

Author contributions: N. Ahmad, A. Javaid, Z. Shaheen and A.H. Khan designed the study. N. Ahmad, A. Javaid and Z. Shaheen collected the data. N. Ahmad analysed the data and wrote the manuscript. A. Javaid, Z. Shaheen and A.H. Khan critically reviewed the manuscript.

\section{References}

1 World Health Organization, End TB Strategy. WHO Treatment Guidelines for Drug-resistant Tuberculosis. Geneva, World Health Organization, 2016. Available from: www.who.int/tb/MDRTBguidelines2016.pdf

2 Van Deun A, Maug AKJ, Salim MAH, et al. Short, highly effective, and inexpensive standardised treatment of multidrug-resistant tuberculosis. Am J Respir Crit Care Med 2010; 182: 684-692. 
3 Piubello A, Harouna SH, Souleymane M, et al. High cure rate with standardised short-course multidrug-resistant tuberculosis treatment in Niger: no relapses. Int J Tuber Lung Dis 2014; 18: 1188-1194.

4 Aung K, Van Deun A, Declercq E, et al. Successful '9-month Bangladesh regimen' for multidrug-resistant tuberculosis among over 500 consecutive patients. Int J Tuber Lung Dis 2014; 18: 1180-1187.

5 Sotgiu G, Tiberi S, D'Ambrosio L, et al. WHO recommendations on shorter treatment of multidrug-resistant tuberculosis. Lancet 2016; 387: 2486-2487.

6 World Health Organization, Global TB Programme. Updated Interim Critical Concentrations for First-line and Second-line DST. www.stoptb.org/wg/gli/assets/documents/Updated\%20critical\%20concentration\%20table_1st\% 20and\%202nd\%20line\%20drugs.pdf May 2012. Date last accessed: September 28, 2016.

7 Zignol M, Dean AS, Alikhanova N, et al. Population-based resistance of Mycobacterium tuberculosis isolates to pyrazinamide and fluoroquinolones: results from a multicountry surveillance project. Lancet Infect Dis 2016; 16: $1185-1192$

8 Varaine F, Guglielmetti L, Huerga $\mathrm{H}$, et al. Eligibility for the shorter multidrug-resistant tuberculosis regimen: ambiguities in the World Health Organization recommendations. Am J Respir Crit Care Med 2016; 194: $1028-1029$.

9 Ahmad N, Javaid A, Basit A, et al. Management and treatment outcomes of MDR-TB: results from a setting with high rates of drug resistance. Int J Tuberc Lung Dis 2015; 19: 1109-1114.

10 Sotgiu G, Tiberi S, D'Ambrosio L, et al. Faster for less: the new "shorter" regimen for multidrug-resistant tuberculosis. Eur Respir J 2016; 48: 1503-1507.

11 Falzon D, Gandhi N, Migliori GB, et al. Resistance to fluoroquinolones and second-line injectable drugs: impact on multidrug-resistant TB outcomes.. Eur Respir J 2013; 42: 156-168.

12 Johnston JC, Shahidi NC, Sadatsafavi M, et al. Treatment outcomes of multidrug-resistant tuberculosis: a systematic review and meta-analysis. PloS One 2009; 4: e6914.

13 Jabeen K, Shakoor S, Malik F, et al. Fluoroquinolone resistance in Mycobacterium tuberculosis isolates from Pakistan 2010-2014: Implications for disease control. Int J Mycobacteriol 2015; 4: 47-48.

14 Ahmad N, Javaid A, Sulaiman SAS, et al. Resistance patterns, prevalence, and predictors of fluoroquinolones resistance in multidrug resistant tuberculosis patients. Braz J Infect Dis 2015; 20: 41-47.

15 Shah SK, Sadiq H, Khalil M, et al. Do private doctors follow national guidelines for managing pulmonary tuberculosis in Pakistan? East Mediterr Health J 2003; 9: 776-788. 